\title{
Commanding Wheelchair in Virtual Reality with Thoughts by Multiclass BCI based on Movement-related Cortical Potentials
}

Mei Lin Chen

Lin Yao

Ning Jiang
University of Waterloo, ON, Canada

University of Waterloo, ON, Canada

University of Waterloo, ON, Canada

\section{Abstract}

Brain-driven wheelchair control is an attractive application in the Brain-Computer Interface (BCI) field. In this research, we designed and validated a virtual wheelchair navigation system controlled by our latest multiclass BCI Menu interface based on a fast brain switch, which provides five commands: move forward, turn left, turn right, move backward, and stop. Preliminary results have shown that subjects can successfully control the wheelchair to hit all targets in the immersive virtual reality (VR) environment. This system proves an avenue to bridge the gap between simulation control in VR environments and real-life wheelchair applications for mobility impairment.

\section{Introduction}

Virtual reality provides a method to immersively mimic the reallife situation. The combination of a BCI system with the immersive virtual environment provides not only the platform for validation of the BCI system's potential performance, but also a training strategy to bridge the gap between laboratory results and real-life applications.

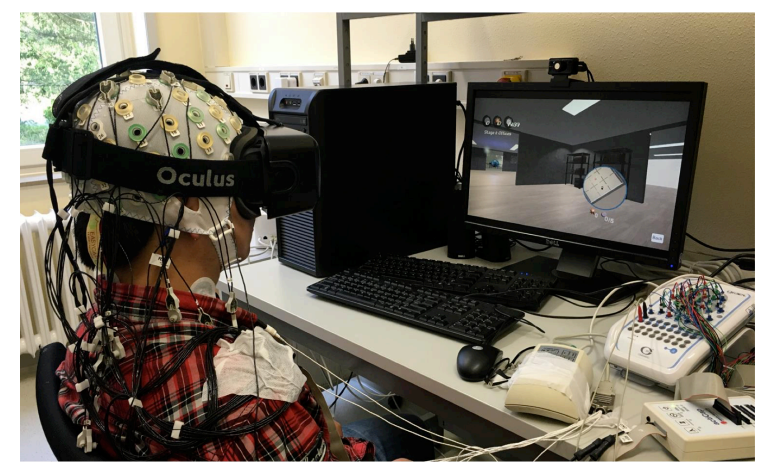

Fig. 1: BCI and VR Systems Setup.

In this study, we designed and validated a virtual wheelchair navigation system, mimicking the real wheelchair in an immersive environment. The multiclass BCI menu based on movement-related cortical potentials (MRCP) fast brain switch was integrated to have an intuitive navigation control $[1,2]$. The VR scene included an apartment with multiple rooms and furniture.

\section{Methods}

As shown in Fig. 1, in the multiclass BCI menu system, four stimulation electrodes were attached around the neck (front, right, back and left). The electrodes were activated clockwise. The inter-stimulus interval was $2 \mathrm{~s}$, and each stimulus lasted $0.2 \mathrm{~s}$, resulting in an 8-s full menu cycle. The brief stimulation burst through an electrode indicated the currently active option to the subject (movement command), and he or she had $2 \mathrm{~s}$ to perform the motor execution task or motor imagery task.

In this way, when the clockwise electrical stimulation started, subjects could select one of the four commands (moving forward, turning left, turning right, moving backward) by generating an MRCP signal at the appropriate time. After that, the wheelchair was commanded to move, and the stimulation was turned off.
When the subject wanted to stop the wheelchair, he or she generated an MRCP signal again. If the command was successful, the wheelchair stopped and at the same time, the BCI menu was activated again to allow the selection of the next movement command. If the subject hit any obstacle (wall, furniture), the wheelchair stopped immediately and the BCI menu was activated. The task for the subject was to navigate the wheelchair from the starting position to the target positions in the virtual apartment, passing through the doors connecting the rooms while avoiding collision.

\section{Results and discussions}
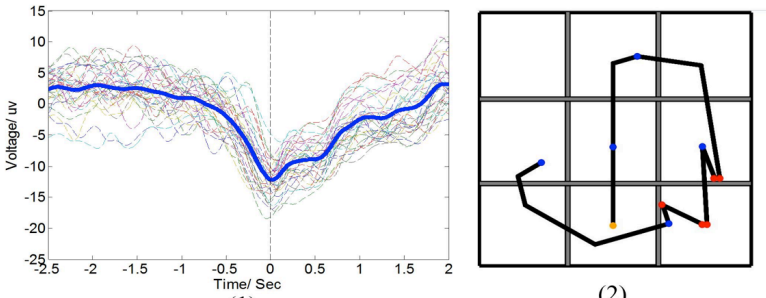

(2)

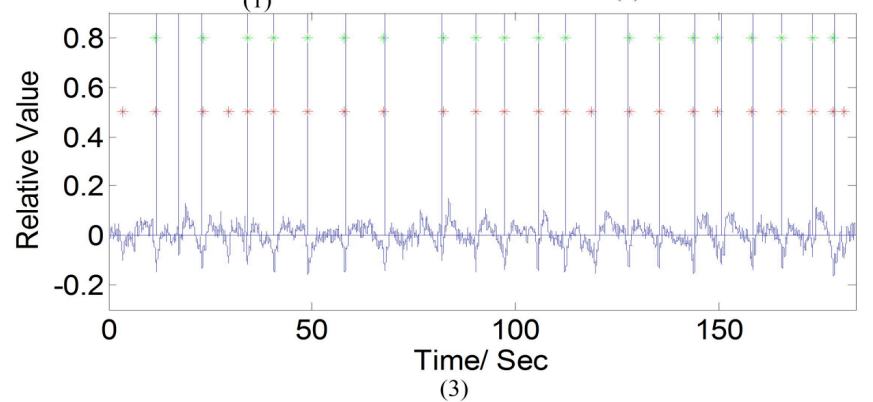

Figure 2: Preliminary Results. (1) MRCP in single-trial, and averaged one as blue line. (2) Trajectory in VR navigation. (3) On-line BCI detection. Red dot indicates the BCI output, vertical line indicates dorsiflexion task as detected by EMG (labels), green dot indicates true detection, blue curves indicates Laplace filtered slow cortical potential between 0.05 to $3 \mathrm{~Hz}$.

Fig. 2 shows the preliminary results of the BCI controlled VR wheelchair navigation. The subject successfully controlled the wheelchair to hit all the targets. Other BCI modalities such as tactile BCI will be integrated to have more controlling commands and reduce the mental loads [3].

\section{Conclusions}

This system proves immersive VR to be a viable platform for $\mathrm{BCI}$ training. It also validates the VR wheelchair navigation system controlled by multiclass BCI based on movement-related cortical potentials, which can be translated into real-world applications to help with mobility impairment.

\section{References}

[1] Xu, R., Jiang, N., Lin, C., Mrachacz-Kersting, N., Dremstrup, K., \& Farina, D. (2014). Enhanced low-latency detection of motor intention from EEG for closed-loop braincomputer interface applications. Biomedical Engineering, IEEE Transactions on, 61(2), 288-296.

[2] Xu, R., Jiang, N., Dosen, S., Lin, C., Mrachacz-Kersting, N., Dremstrup, K., \& Farina, D. (2016). Endogenous Sensory Discrimination and Selection by a Fast Brain Switch for a High Transfer Rate Brain-Computer Interface. Neural systems and Rehabilitation Engineering, IEEE Transactions on, DOI: 10.1109/TNSRE.2016.2523565

[3] Yao, L., Meng, J., Zhang, D., Sheng, X., \& Zhu, X. (2013). Selective sensation based brain-computer interface via mechanical vibrotactile stimulation. PloS one, 8(6), e64784. 